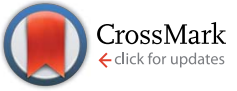

Cite this: RSC Adv., 2017, 7, 12842

Received 12th January 2017

Accepted 15th February 2017

DOI: $10.1039 / \mathrm{c} 7 \mathrm{ra00500h}$

rsc.li/rsc-advances

\section{Experimental evidence of electronic polarization in a family of photo-ferroelectrics}

\author{
Hitesh Borkar, ${ }^{\text {ab }}$ Vaibhav Rao, ${ }^{\text {acg }}$ M. Tomar, ${ }^{d}$ Vinay Gupta, ${ }^{\text {e }}$ J. F. Scott ${ }^{f}$ \\ and Ashok Kumar*ab
}

\begin{abstract}
We report a family of ferroelectric materials which exhibit optical control of dielectric constant, polarization, and conductivity at ambient conditions. The evolution of photo-induced charge carriers under illumination of weak monochromatic light of wavelength $\lambda=405 \mathrm{~nm}$ developed electronic polarization that reorients or enhances the ionic polarization in the direction of the applied electric field, hence significantly increasing the net polarization. The presence of electronic polarization in perovskites, especially $\mathrm{ABO}_{3}$ oxides, and the contribution to the ionic polarization, is a matter of debate among ferroelectric community; however, recent experimental evidence proved that electronic polarization contributes to overall polarization in different ways: if it is additive, it will act along the direction of ionic polarization; and if it is subtractive, it will act in the opposite direction. We report a newly developed lead-based ferroelectric family $\mathrm{Pb}_{1-x}\left(\mathrm{Li}_{0.5} \mathrm{Bi}_{0.5}\right)_{x}\left(\mathrm{Zr}_{0.2} \mathrm{Ti}_{0.8}\right) \mathrm{O}_{3}$ (PLBZT) for $x=0.3,0.4,0.5$, which have been successfully synthesized by a conventional solid state reaction route. The $\mathrm{X}$-ray diffraction patterns were recorded at room temperature and refined with Rietveld parameters which confirm its tetragonal structure with P4mm space group symmetry. The Raman spectra, dielectric properties and leakage currents are analyzed. The current conduction mechanisms obey bulk limited Poole-Frenkel model and provide optical dielectric constant similar to theoretical value to lead zirconate titanate.
\end{abstract}

\section{Introduction}

Several years ago, V. M. Fridkin proposed mechanisms and related phenomena for possible conversion of light to electricity using bulk ferroelectrics, i.e. "photo-ferroelectrics". ${ }^{1}$ In the following decades further development has been taken place on ferroelectric photovoltaic (PV) materials and devices, ${ }^{2,3}$ mostly centered, however, around the understanding of basic physics. A good recent example of the controversy in assessing electronic contributions (as contrasted with ionic contributions) to ferroelectricity is afforded by the work on $\mathrm{K}_{3} \mathrm{Fe}_{5} \mathrm{~F}_{15}$, where the experiments of Blinc et al. ${ }^{\mathbf{4}-6}$ showed no electronic contribution, but subsequent DFT theory by Yamauchi and Picozzi ${ }^{7}$ incorrectly inferred an electronic (non-ionic) origin.

${ }^{a}$ CSIR-National Physical Laboratory, Dr K. S. Krishnan Marg, New Delhi 110012, India.E-mail: ashok553@nplindia.org

${ }^{b}$ Academy of Scientific and Innovative Research (ACSIR), CSIR-National Physical Laboratory (CSIR-NPL) Campus, Dr K. S. Krishnan Road, New Delhi 110012, India 'Solar and Alternative Energy, Amity University, Jaipur, Rajasthan, 302006, India ${ }^{d}$ Department of Physics, Miranda House, University of Delhi, Delhi 110007, India ${ }^{e}$ Department of Physics and Astrophysics, University of Delhi, Delhi 110007, India ${ }^{f}$ Department of Chemistry and Department of Physics, University of St. Andrews, St. Andrews KY16 ST, UK

${ }^{g}$ Ultrasound Research Institute, Kaunas University of Technology, Barsausko st. 59, Kaunas LT-51368, Lithuania
Photo-ferroics are a particular class of materials where phenomena, the ferroelectric effect and the photoelectric or photovoltaic effect are intimately linked. These two effects are somewhat incompatible in nature since high quality ferroelectrics must be electrical insulators in order to withstand large applied voltages or fields, whereas photovoltaic devices need high open-circuit voltage and short-circuit current. A big advantage of photo-ferroelectrics compared to conventional silicon or organic photovoltaic devices is their directionality: polarization dictates the direction of photocurrents. The photoferroic systems develop a complex relation between the ferroelectric phase stability (including domain size and distribution) and photo-response; these photo-generated charge carriers (electrons and holes) in relatively high concentration affect the free energy close to ferroelectric transition (Curie point). The nature and magnitude of phase transition can be significantly altered by the photo-excited electron sub-system.

In general it has been observed that the presence of photoexcited charge carriers considerably changes the polarization hysteresis $^{8}$ and shifts the ferroelectric phase transition temperature towards lower temperatures.9,10 Photo-assisted polarization hysteresis may increase or decrease depending on screening effects, population of defect centers, trap-filling, and neutralization of depolarization fields. The Curie temperatures $\left(T_{\mathrm{c}}\right)$ were considerably lowered as observed long ago in SbSI, and the shifts in $T_{\mathrm{c}}$ were proportional to the carrier 
concentrations in the matrix. ${ }^{\mathbf{1 1}}$ The presence of charge carriers may cause structural deformation where the unit cell volume gets affected by generation of charge carriers during the phase transition. The effective permittivity has strong dependence on the concentration of carriers. Readers are referred to V. M. Fridkin's seminal texts for the physical manifestations of the photo-ferroic effect. ${ }^{\mathbf{1 , 1 2}}$

Recently Dawber et al. ${ }^{13}$ explained the possible origin and direction of electronic polarization in perovskite $\mathrm{BaTiO}_{3}$. They proposed the change in the electronic weight due to displacement of central cations and 2 p electron orbitals for oxygen ions which in turn modifies the ionic polarization. This effect may be more significant if one can change the electron population by any other means. For example, under illumination of light, a significant amount of photo-generated charge carriers develop in the matrix which notably alters the $2 p$ electron orbitals and hence can produce giant electronic polarization in the direction of ionic polarization. The $\mathrm{HoMn}_{2} \mathrm{O}_{5}$ crystal polarization depends on the spin direction, which leads to electronic polarization opposite to the ionic polarization of the system. ${ }^{\mathbf{1 4}}$ The electronic polarization vector may be in the direction of ionic polarization or in the opposite direction; this depends on the electron transfer contribution, hybridization of electronic orbitals, and spectral weight of electron density. A giant electronic polarization was experimentally observed in molecular ferroelectric crystals; their magnitudes were an order of magnitude higher than the ionic polarization. Kobayashi et al. performed polarization experiments on the organic molecular crystal tetrathiafulvalene $p$-chloranil (TTF-CA) that showed giant electronic polarization below $81 \mathrm{K.}^{15}$

The electron-hole pairs (excitons) are tightly bound in many ferroelectrics, but can be separated at the domain walls by inbuilt electric field or under illumination of light or application of external electric fields. These free charge carriers significantly alter the depolarization fields, which in turn change the net polarization of the system. The effective collection of charge carriers also depends on the choice and design of electrodes. The proper selection of low work-function electrodes tune the barrier height of the Schottky contact near metalferroelectric interface and make a balance between requirements of large barrier height for switchable polarization and ohmic contact for high photovoltaic effects. ${ }^{\mathbf{1 6}, 17}$ The unconventional bulk ferroelectric photovoltaic effect was extensively explored in early 1970's and explained on the basis of noncentrosymmetric nature of the crystals. ${ }^{12,18}$ However, with the advent of sophisticated thin-film fabrication techniques, ferroelectric-photovoltaic effects and low bandgap ferroelectric materials received great attention due to strong coupling of polarization and light. ${ }^{19-21}$

Among the ferroelectrics families lead zirconate titanate $\mathrm{PbZr}_{x} \mathrm{Ti}_{1-x} \mathrm{O}_{3}$ (PZT) found a special place due to its optimal properties, such as large fatigue-free polarization, ${ }^{22}$ piezoelectricity, ${ }^{23}$ and good electro-optic effect. ${ }^{24,25}$ These properties are related to polarization behavior under the influence of external electric field. ${ }^{26-28}$ Since the discovery of PZT, extensive research has been carried out on substitution of various types of di- and tri-valence cations on lead and zirconia/titanate sites. Some of these substitutions were very successful and led to development of device quality materials with improved optical, ferroelectric, dielectric and leakage properties. The substitutions also change the crystal properties from ferro- to antiferro- to para-electric and modify the ferroelectric phase transition temperatures depending on the substitution elements and their magnitude. We have substituted optically active $\mathrm{Li}^{1+}$ and $\mathrm{Bi}^{3+}$ cations together to provide necessary charge neutrality for the formation of single phase light sensitive PLBZT systems. Here we report a novel family of optically active modified PZT electroceramics with $30-50 \%$ substitution of $\mathrm{Pb}^{4+}$ by $\mathrm{Bi}^{3+}$ and $\mathrm{Li}^{1+}$ cations. The functional properties depend on substitution of $\mathrm{Li}$ and Bi cations in PZT matrix.

\section{Experimental details}

Bulk polycrystalline ceramics pellets of $\mathrm{Pb}_{1-x}\left(\mathrm{Li}_{0.5} \mathrm{Bi}_{0.5}\right)_{x}\left(\mathrm{Zr}_{0.2} \mathrm{Ti}_{0.8}\right)$ $\mathrm{O}_{3}$ (PLBZT) for $x=0.3,0.4,0.5$ were synthesized by a solid state reaction technique. The oxide and carbonate powders of $\mathrm{PbO}$, $\mathrm{Li}_{2} \mathrm{CO}_{3}, \mathrm{Bi}_{2} \mathrm{O}_{3}, \mathrm{ZrO}_{2}$, and $\mathrm{TiO}_{2}$ from Sigma-Aldrich (purity < 99.9\%) were mechanically mixed and ground for several hours to prepare homogeneous precursors. An extra amount (5\%) of $\mathrm{PbO}, \mathrm{Li}_{2} \mathrm{CO}_{3}$, $\mathrm{Bi}_{2} \mathrm{O}_{3}$, was taken to maintain the stoichiometry during high sintering temperature. The green powders with proper stoichiometry were mechanically milled in isopropyl alcohol (IPA) in agate mortar for $2 \mathrm{~h}$ and calcined at $850{ }^{\circ} \mathrm{C}$ for $10 \mathrm{~h}$. These calcined powders were re-ground and mixed with $10 \mathrm{wt} \%$ polyvinyl alcohol (PVA) solution in order to prepare circular disc-shaped pellets for further analysis. The binder-mix granulated powders were shaped into discs of $13 \mathrm{~mm}$ diameter and 1-1.5 mm thickness under uniaxial pressure of 5-6 tons per square inch. Finally these pellets were sintered in air at $1200{ }^{\circ} \mathrm{C}$ for $4 \mathrm{~h}$ to achieve $94-97 \%$ of theoretical density. ${ }^{29}$ The powder X-ray diffraction (XRD) (Bruker AXS D8 Advance X-ray diffractometer) using the Cu- $\mathrm{k}_{\alpha}\left(\mathrm{k}_{\alpha}=1.5405\right.$ A) monochromatic radiation, in the $2 \theta$ range between $20^{\circ}$ and $60^{\circ}$ was used to determine the phase purity, crystal structure, lattice constant and strain. Grain growth, surface morphology, and percentage of elemental compositions on sintered pellets were carried out using a scanning electron microscope (SEM, Zeiss EVOMA-10) and energy-dispersive X-ray spectroscopy (EDX). The Xray photoemission spectroscopy (XPS) has been utilized to check the valance states of various constituents. During the scan process, core levels C 1s spectrum has maintained at $284.5 \mathrm{eV}$. Subtle structural distortions, local defects and different ordering states in perovskite structures were studied using room temperature Raman spectra via a Renishaw Via Reflex Raman spectrometer, with an excitation source of $514.5 \mathrm{~nm}(2.78 \mathrm{eV})$ with a resolution of less than $1.0 \mathrm{~cm}^{-1}$. For all electro-optic measurements samples were mechanically polished to make a thin slab of $0.3-0.5 \mathrm{~mm}$. Further transparent conductive electrodes of ITO (Indium Tin Oxide) layer were coated using RF magnetron sputtering on the top surface and silver as bottom electrode. The ITO was chosen as top transparent electrode to transmit light over the ferroelectric surface, and to produce a good barrier height at the ferroelectric interface. The selection of electrodes is important for transport properties and good ferroelectric properties with one interface as a Schottky contact. ${ }^{30-32}$ Thus, careful attention is needed in 
selection of electrode materials to achieve efficient and/or switchable photo-ferroelectric devices. To neutralize the pinning effects, trapping of charges at grain boundaries and filling of oxygen vacancies with free charge carriers in the matrix, these ceramics were electrically poled at room temperature by applying a dc field of $40 \mathrm{kV} \mathrm{cm}{ }^{-1}$ for $3-5 \mathrm{~h}^{33}$ Photo-charge carriers were generated on the top surface and also inside the bulk under illumination of a white light source $(40 \mathrm{~mW})$ and also with a monochromatic u.v. laser having wavelength $405 \mathrm{~nm}(30 \mathrm{~mW})$. Temperature dependent dielectric properties were studied using a LCR meter (HIOKI-3532-50) at an oscillating amplitude of $0.5 \mathrm{~V}$ under dark and under illumination of monochromatic laser light of $405 \mathrm{~nm}$ wavelength at various frequencies ( $1 \mathrm{kHz}$ to $1 \mathrm{MHz})$ in the temperature range from $25{ }^{\circ} \mathrm{C}$ to $450{ }^{\circ} \mathrm{C}$. Polarization versus electric field $(P-E)$ hysteresis loops and leakage current were measured by using a Radiant Ferroelectric Tester under both dark and illuminated conditions.

\section{Results and discussion}

\section{(a) X-ray diffraction patterns}

Fig. 1(a), (b) and (c) show room temperature X-ray diffraction patterns of PLBZT for $x=0.3,0.4,0.5$, respectively. All of the Braggs peaks were indexed carefully and correspond to a tetragonal crystal structure with small variation in lattice constants. Rietveld refinement parameters are shown in Table 1 that indicate increase in in-plane lattice constant $(a)$ and decrease in out-of-plane lattice constant $(c)$ with increase in $\mathrm{Li}$ and $\mathrm{Bi}$ cation compositions which belong to space group symmetry $P 4 \mathrm{~mm}$. Fitting parameters for $x=0.3$ and 0.4 were in good agreement with tetragonal structure with goodness of fit of $\chi^{2}=1.90$; but with further increase in concentration of $\mathrm{Bi}$ and $\mathrm{Li}$ cations $(x=0.5)$, a distortion develops in crystal structure, specifically on the perovskite $\mathrm{Pb}$-site, which in turn creates some secondary and impurity phases. The XRD peak close to 28.4 Braggs angle represents the secondary phase of $\mathrm{Pb}_{3} \mathrm{Bi}_{4}$ $\mathrm{Ti}_{6} \mathrm{O}_{21}$ alloys (*JCPDS file no. 35-0007) and it was found in the range of 1 to $1.5 \%$, where the composition with $x=0.5$ shows highest $1.5 \%$ of impurity phase. The amount of impurity phase is negligible that hardly affects any functional properties. This much amount of impurity phase is very common in multi components ceramics which is very unlikely to avoid. These results indicate coexistence of $\mathrm{Bi}^{3+}$ and $\mathrm{Li}^{1+}$ cations on $\mathrm{Pb}$-site to replace some of the $\mathrm{Pb}^{2+}$ cations. ${ }^{34}$ These results suggest chemical and compositional homogeneity of $\mathrm{Zr}^{4+} / \mathrm{Ti}^{4+}$ cations at B-site and $\mathrm{Pb}^{2+} /\left(\mathrm{Bi}^{3+} / \mathrm{Li}^{1+}\right)$ at A-site. Surface morphology and final compositions of each system of the sintered ceramics were analyzed using large-area SEM images and elemental mapping, respectively. As depicted in the inset of Fig. 1(a)-(c) for respective ceramics, a granular structure is revealed with little porosity for all the compositions (inset image represents the compositions as in the XRD). These images clearly demonstrate that the average grain size increases progressively with the increase of percentage of the $\mathrm{Bi} / \mathrm{Li}$ cations. It can be observed for all compositions that a granular structure with well-defined grains and grain boundaries with few voids results. The images show that the surfaces are homogeneous and densely packed, and

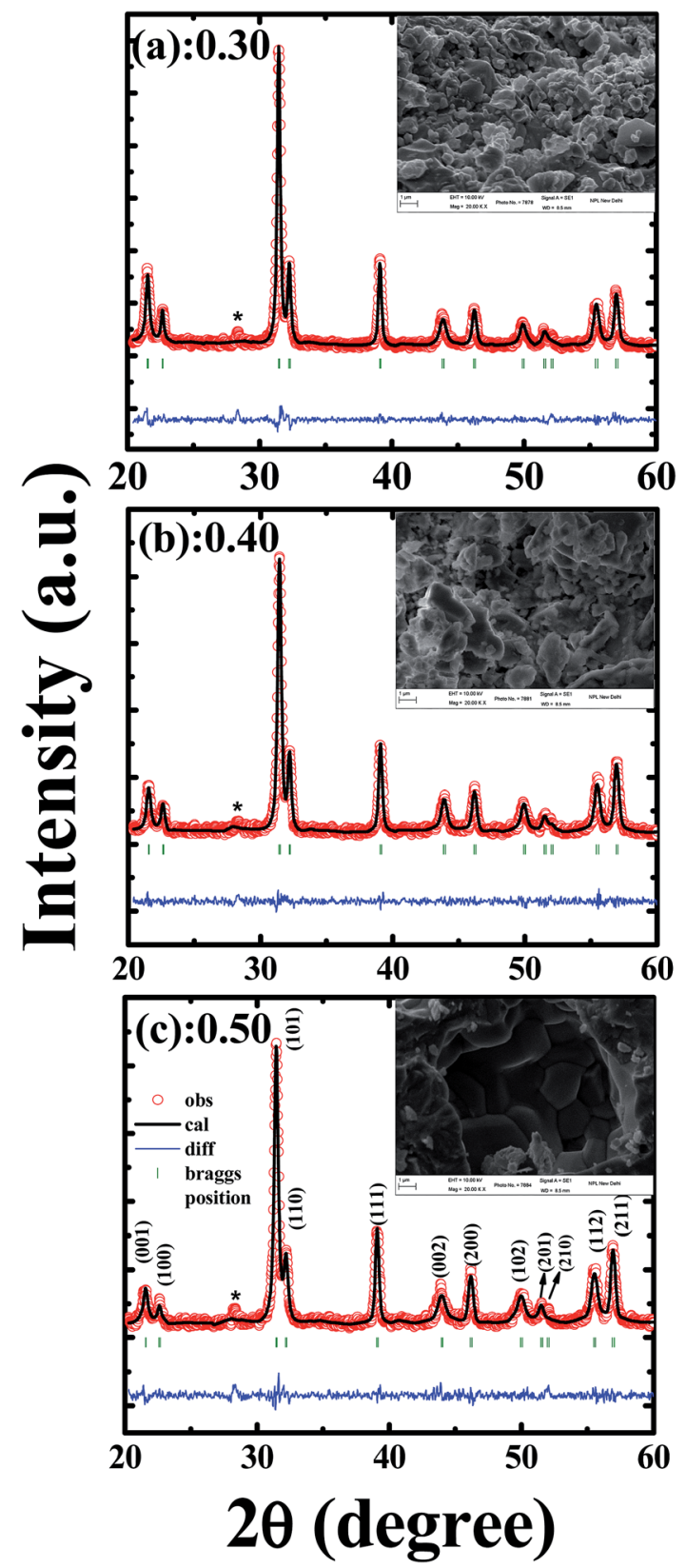

Fig. 1 Rietveld refine $X$-ray diffraction (XRD) pattern for polycrystalline ceramics $\mathrm{Pb}_{1-x}\left(\mathrm{Li}_{0.5} \mathrm{Bi}_{0.5}\right)_{x}\left(\mathrm{Zr}_{0.2} \mathrm{Ti}_{0.8}\right) \mathrm{O}_{3}$ for $x=0.3$ (a), 0.4 (b), 0.5 (c) fitted with space group model $P 4 \mathrm{~mm}$. Small amount (1-1.5\%) of secondary phase mark as * for $\mathrm{Pb}_{3} \mathrm{Bi}_{4} \mathrm{Ti}_{6} \mathrm{O}_{21}$ alloys (JCPDS file no. 350007). Inset SEM micrograph of corresponding composition.

their large area EDX analysis suggests the compositional percentage of each initial and final precursor elements are well within the errors limits $( \pm 10 \%)$ of SEM-EDX. Light element such as lithium cannot be detected by SEM-EDX. Table 2 shows detailed compositional analysis of various elements.

\section{(b) Raman spectroscopy}

Raman spectroscopy is a nondestructive and highly sensitive local probe technique which identifies and investigates the local as well as global crystal structures, structural phase transitions, 
Table 1 Refined structural parameters of PLBZT for $x=0.3,0.4$, and 0.5 using tetragonal structure

\begin{tabular}{llll}
\hline Refine parameters & $x=0.3$ & $x=0.4$ & $x=0.5$ \\
\hline$a(\AA)$ & $3.925(29)$ & $3.929(86)$ & $3.931(12)$ \\
$b(\AA)$ & $3.925(29)$ & $3.929(86)$ & $3.931(12)$ \\
$c(\AA)$ & $4.126(88)$ & $4.124(45)$ & $4.118(80)$ \\
Structure & Tetragonal & Tetragonal & Tetragonal \\
Space group & $P 4 m m$ & $P 4 m m$ & $P 4 m m$ \\
$R_{\text {Braggs }}$ & 2.17 & 2.047 & 2.87 \\
$R_{\mathrm{f}}$ Factor & 1.57 & 1.832 & 3.24 \\
$R_{\mathrm{p}}$ & 14.15 & 19.0 & 20.0 \\
$R_{\mathrm{w}}$ & 15.4 & 19.02 & 19.8 \\
$R_{\exp }$ & 11.36 & 14.11 & 13.19 \\
$\chi^{2}$ & 1.90 & 1.85 & 2.39 \\
& & &
\end{tabular}

impurity phases, stress, and nano-scale alien phases. Previous attempts were made to explain the Raman modes of Ti-rich lead-zirconate-titanate based perovskite oxides. ${ }^{7,35,36}$ We have explained the Raman spectra of PLBZT ceramics for $x=0.3,0.4$, 0.5 in context of earlier assigned Raman modes, as can be seen in Fig. 2(a) perovskite structure with general formula $\mathrm{ABO}_{3}$ consists of two sublattices: a covalent-bonded lattice formed of octahedral $\mathrm{BO}_{6}$ sharing their oxygen atoms, and an A-cation sublattice with coulomb interaction. For PLBZT ceramics, the A-sublattices are occupied by $\mathrm{Pb}^{2+} / \mathrm{Bi}^{3+} / \mathrm{Li}^{1+}$ cations, and $\mathrm{B}$-site $\mathrm{Zr}^{4+} / \mathrm{Ti}^{4+}$ ions are randomly distributed in the $\mathrm{ABO}_{3}$ structure.

Raman spectra for PLBZT series were analyzed using the Lorentzian function-based damped harmonic oscillator model (DHO) for these compositions, as shown in Fig. 2(b)-(d). ${ }^{37-39}$ Based on group theory, pure tetragonal $\mathrm{PbTiO}_{3}$ system with space group $P 4 \mathrm{~mm}$ has been assigned for optical-phonons $\mathrm{T}_{1 \mathrm{u}}$ and $\mathrm{T}_{2 \mathrm{u}}$ modes which splits into two modes as $\mathrm{A}_{1}+\mathrm{E}$ and $\mathrm{B}_{1}+\mathrm{E}$ (silent), respectively. All these modes are both Raman and infrared active. These modes are leveled in sequence 1, 2, 3 from low to high frequency. ${ }^{39-43}$ A large number of Raman spectroscopy work has been carried out on Ti rich-PZT systems, its Raman bands have already been defined and assigned. Present systems possess large amount of $\mathrm{Bi}$ and Li substitution at A-site that leads to diffuse Raman spectra. All possible Raman modes are fitted with damped harmonic oscillator model (DHO). The fitted data are presented in the Fig. 2(b)-(d) for $x=0.3,0.4,0.5$, respectively. Raman spectra show nine dominant Raman bands, centered at around $151 \mathrm{~cm}^{-1} \mathrm{E}\left(\mathrm{LO}_{1}\right), 224 \mathrm{~cm}^{-1} \mathrm{E}\left(\mathrm{TO}_{2}\right)$, $280 \mathrm{~cm}^{-1}\left(\mathrm{~B}_{1}+\mathrm{E}\right), 345 \mathrm{~cm}^{-1} \mathrm{~A}_{1}\left(\mathrm{TO}_{2}\right), 452 \mathrm{~cm}^{-1} \mathrm{E}\left(\mathrm{LO}_{2}\right), 514 \mathrm{~cm}^{-1}$ $\mathrm{E}\left(\mathrm{TO}_{3}\right), 630 \mathrm{~cm}^{-1} \mathrm{~A}_{1}\left(\mathrm{TO}_{3}\right), 712 \mathrm{~cm}^{-1} \mathrm{E}\left(\mathrm{LO}_{3}\right)$ and $750 \mathrm{~cm}^{-1}$ $\mathrm{A}_{1}\left(\mathrm{LO}_{3}\right)$, respectively. ${ }^{40,41}$ These Raman bands have slightly different frequency positions from those observed in Ti-rich PZT $(20: 80) 3^{39-43}$ however, their magnitudes are quite different, which clearly supports the model of large amount of substitution of $\mathrm{Bi} / \mathrm{Li}$ at A-sites. If we look carefully at the lowfrequency Raman bands, it can be clearly seen that the intensities of Raman bands at $151 \mathrm{~cm}^{-1}$ and $224 \mathrm{~cm}^{-1}$ (for PZT $20: 80$ around $220 \mathrm{~cm}^{-1}$ ) diminish with increase in $\mathrm{Bi} / \mathrm{Li} \%$, and the magnitude at $280 \mathrm{~cm}^{-1}$ significantly increases compared to the lower Raman bands with increase in $\mathrm{Bi} / \mathrm{Li}$ substitution. Raman modes are fitted with DHO model ${ }^{39,41}$ and their full width at half maximum (FWHM) and Raman bands are obtained. The FWHM of the most intense $\mathrm{E}\left(\mathrm{TO}_{2}\right)$ mode is found to be $25.28 \mathrm{~cm}^{-1}, 20.92 \mathrm{~cm}^{-1}, 18.91 \mathrm{~cm}^{-1}$ and for $\left(\mathrm{B}_{1}+\mathrm{E}\right)$ mode $19.35 \mathrm{~cm}^{-1}, 18.14 \mathrm{~cm}^{-1}, 18.07 \mathrm{~cm}^{-1}$ for $x=0.3,0.4,0.5$, respectively. These results revealed the information regarding broadening of peak as increase the doping concentration. It is found that substitution of $\mathrm{Bi}$ and $\mathrm{Li}$ cations affect the Raman linewidth, and the FWHM value is inversely proportional to the phonon lifetime. ${ }^{42}$

It is well known that Bi substitution at A-site significantly increases the intensity of the $\mathrm{B}_{1}+\mathrm{E}$ mode doublet, as seen in Raman spectra of $\mathrm{BiFeO}_{3}{ }^{43}$ This phenomenon may be related to short/long-range intrinsic disorder, where the ions are displaced from their high mean symmetry positions, giving rise to the local electric dipoles. ${ }^{44,45}$ The variation of the Raman bands in the low-frequency region is attributed to the substitution of A-site elements of crystal structure and stretching/lattice modes. The variation in the intensity of the various peaks below $400 \mathrm{~cm}^{-1}$ (from Fig. 2) could be a result of the substitution of $\mathrm{Bi}$ and $\mathrm{Li}$ on the $\mathrm{Pb}$-site sublattice. The presence of three cations $(\mathrm{Pb} / \mathrm{Li} / \mathrm{Bi})$ causes difficulty in analyzing effects of mass and ionic radii (all three having similar ionic radii) on lowfrequency Raman modes. Overlapped Raman spectra of the specimens are mainly due to the random distribution of $\mathrm{Pb}, \mathrm{Bi}$, Li cations which break the wave vector selection rule $(k=0)$ and permit phonons from the entire Brillouin zone to become Raman-active. Three Raman active modes are observed for the medium frequency ranges $\left(300-550 \mathrm{~cm}^{-1}\right)$ which represent oxygen ion vibration and dielectric leakage due to different $\mathrm{A}$

Table 2 Stoichiometric comparison of initial ingredients and final product from Energy Dispersive X-ray element analysis (EDX)

\begin{tabular}{|c|c|c|c|c|c|c|}
\hline Elements & Stoichiometric calculated & Obs. from EDX & $\begin{array}{l}\text { Stoichiometric } \\
\text { calculated }\end{array}$ & $\begin{array}{l}\text { Obs. from } \\
\text { EDX }\end{array}$ & $\begin{array}{l}\text { Stoichiometric } \\
\text { calculated }\end{array}$ & $\begin{array}{l}\text { Obs. from } \\
\text { EDX }\end{array}$ \\
\hline $\mathrm{Pb}$ & 44.44 & 50.23 & 39.78 & 46.91 & 33.14 & 35.67 \\
\hline $\mathrm{Bi}$ & 20.07 & 8.09 & 26.75 & 12.94 & 33.42 & 20.05 \\
\hline $\mathrm{Li}$ & \multicolumn{6}{|c|}{ Not in the range of experimental limits } \\
\hline $\mathrm{O}$ & 15.37 & 21.38 & 15.36 & 16.75 & 15.35 & 20.17 \\
\hline
\end{tabular}




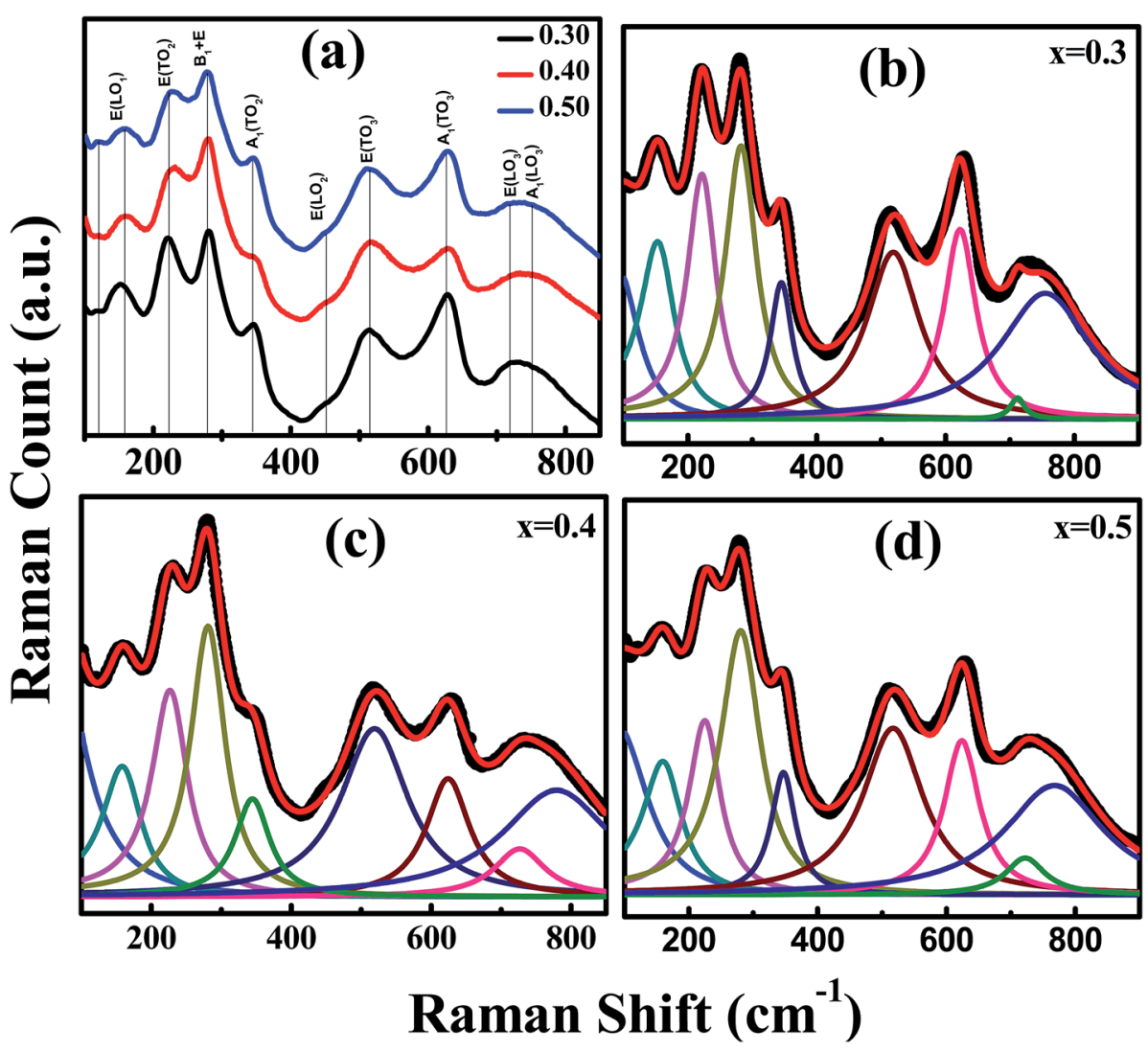

Fig. 2 (a) Room-temperature Raman spectra for PLBZT ceramics with different $x=0.3,0.4,0.5$ of Bi and Li constituents. Spectral deconvolution for a PLBZT content of $x=0.3$ (b), 0.4 (c), 0.5 (d) at room temperature. The spectra were deconvolved based on nine Gaussian-Lorentzian modes and the assignment of the spectral modes to particular lattice vibrations.

and B-site cations. Three high-frequency Raman modes $\mathrm{A}_{1}\left(\mathrm{TO}_{2}\right), \mathrm{E}\left(\mathrm{LO}_{2}\right)$, and $\mathrm{E}\left(\mathrm{TO}_{3}\right)$ seem to be unchanged, whereas phonon modes $\mathrm{A}_{1}\left(\mathrm{TO}_{3}\right)$ undergo an significant change in intensity as a function of $\mathrm{Zr} / \mathrm{Ti}$ concentration. Although the $\mathrm{Zr}$ / Ti composition is constant for all three compositions, the spectral weight of $\mathrm{Bi} / \mathrm{Li}$ substitution at $\mathrm{Pb}$-site significantly alters the high-frequency phonon positions and intensity. ${ }^{7}$

\section{(c) X-ray photon spectroscopy}

Fig. 3(a)-(f) shows the Pb, Li, Bi, Zr, Ti and O elements XPS corelevel spectra of PLBZT $(x=0.4)$ bulk ceramic samples. We have chosen this particular composition for XPS analysis, since it illustrates the best coupling of polarization under illumination of light. The binding energy (B.E) of all the spectra has been explained while measuring C 1s core levels B.E at $284.5 \mathrm{eV}$ as shown in the inset Fig. 3(f). The core-level XPS spectra of $\mathrm{Pb} 4 \mathrm{f}_{7 / 2}$ and $4 \mathrm{f}_{5 / 2}$ were found at $\sim 137.8$ and $\sim 142.7 \mathrm{eV}$, respectively and their difference in binding energy for spin-orbit peaks is about $4.9 \mathrm{eV}$ suggests that $\mathrm{Pb}$ is in a single valence state with $\mathrm{Pb}^{2+}$ ion valency ${ }^{46}$ Fig. 3 (b) shows the core level spectra of $\mathrm{Li} 1 \mathrm{~s}$ which is diffused, broad and weak in nature. It is due to very small amount of Li concentration in PLBZT $(x=0.4)$ and small crosssection of Li ions that makes it difficult to interact with incident X-ray photon. The sharp peak of $\mathrm{Ti} 3 \mathrm{~s}$ also shadowed the lattice site of Li cations generally mentioned at 54.6 to $55.6 \mathrm{eV}$ in literature; however, it is difficult to identify the interstitial and lattice occupancy of $\mathrm{Li}$ ions due to broad hump in the range of 49 to $55 \mathrm{eV}$. The lattice occupancy of Li ions depends on the chemical potential of substitution site. ${ }^{47-49}$ The Bi $4 \mathrm{f}_{7 / 2}$ and $4 \mathrm{f}_{5 / 2}$ spectra display well defined singlet peaks located at $\sim 158.5$ and $\sim 163.7 \mathrm{eV}$, respectively. The binding energy difference between the spin-orbit peaks is about $5.4 \mathrm{eV}$ suggests that $\mathrm{Bi}$ is in a single valence state with $\mathrm{Bi}^{3+}$ ion valency. ${ }^{50-52} \mathrm{~A}$ broad and diffused Ti $2 \mathrm{p}_{3 / 2}$ peak is obtained near $457.8 \mathrm{eV}$ suggests most of the Ti cations are in +4 states. The $\mathrm{Zr} 3 \mathrm{~d}_{5 / 2}$ and $3 \mathrm{~d}_{3 / 2}$ illustrate well defined peaks located at $\sim 181.5 \mathrm{eV}$ and $\sim 183.9 \mathrm{eV}$, respectively with a weak hump near $180 \mathrm{eV}$. The origin of hump near $\mathrm{Zr}$ peaks is unknown which requires further systematic study. O 1s core level spectra have been fitted using two Gaussian components corresponding to oxygen signal from lattice (bulk) and surface contaminants $\left(\mathrm{OH}^{-}\right.$and/or $\left.\mathrm{C}-\mathrm{O}\right) .{ }^{54}$

\section{(d) Dielectric properties under illumination of light}

The room temperature dielectric properties over a wide range of frequency, i.e. $1 \mathrm{kHz}$ to $1 \mathrm{MHz}$ for PLBZT ( $x=0.3,0.4$ and 0.5 ), under dark and monochromatic laser light are shown in Fig. 4(a)-(f). Under illumination of monochromatic light a significant enhancement in the dielectric constant and loss tangent were observed for each system, especially for $x=0.4$ (Fig. 4). The magnitude of dielectric constant and tangent loss 


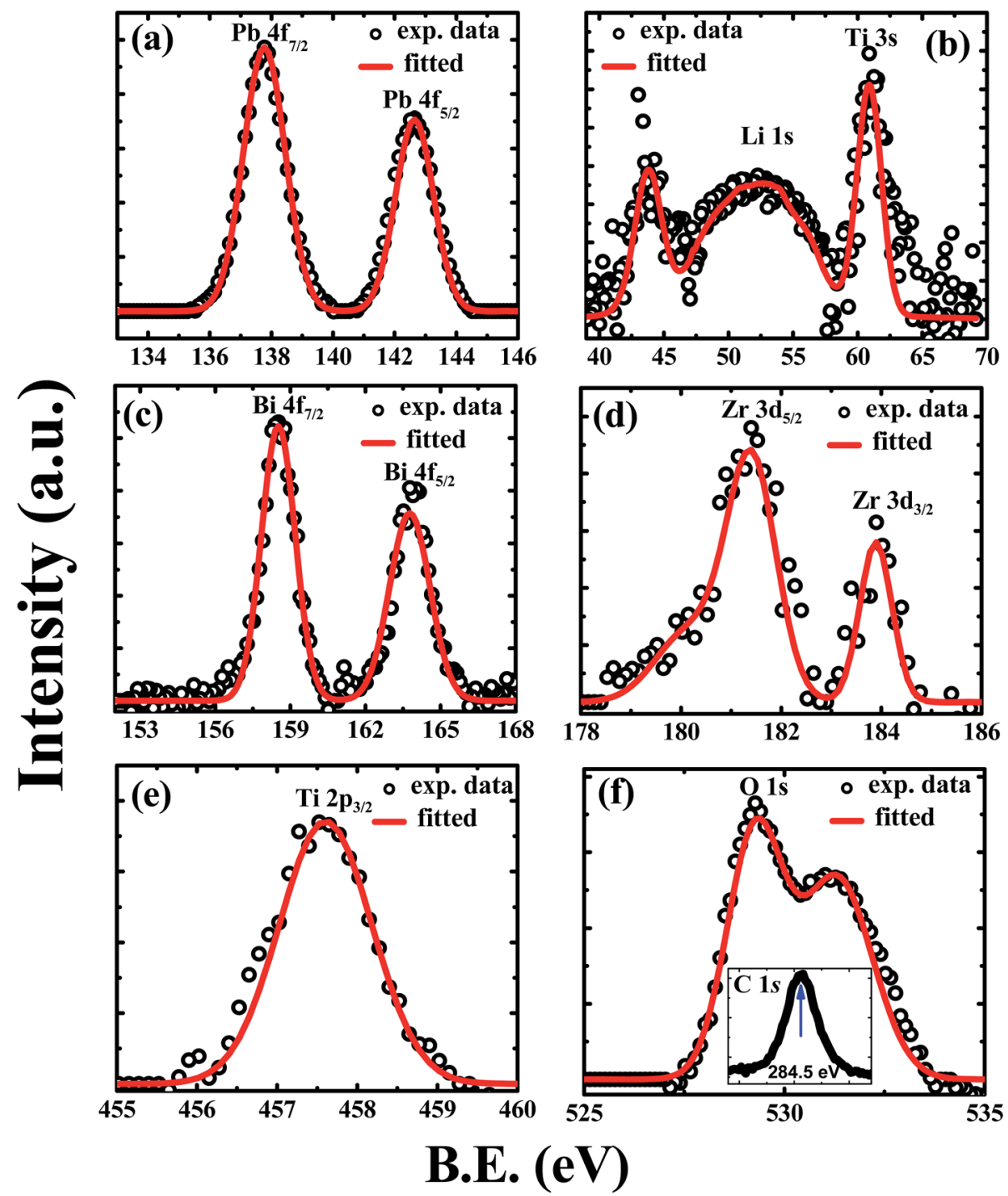

Fig. 3 XPS scan spectra of (a) Pb $4 f$ core level, (b) Li 1s core level, (c) Bi $4 f$ core level, (d) Zr 3d core level, (e) Ti 2p core level, and (f) O 1s core level, (inset $\mathrm{C}$ 1s core level).

decreased with increase in Bi and Li ion substitution; however, the illumination of light significantly increases the values of dielectric constant with nearly the same tangent loss $(\sim 5 \%)$. The dielectric constant and temperature hysteresis $\left(\Delta T_{\mathrm{h}}\right)$ are related phenomenon and follow a relation $\varepsilon\left(T_{\mathrm{c}}\right) \Delta T_{\mathrm{h}} \cong C$. If we suppose electrons do not change the Curie-Weiss constant $C$, then it all depends on temperature hysteresis

$$
\Delta T_{\mathrm{h}} \cong T_{\mathrm{c}}-T_{0}=\frac{1}{8 \pi} C \frac{\beta^{2}}{\gamma}
$$

where $T_{\mathrm{c}}$ is phase transition temperature; $T_{0}$, the Curie-Weiss temperature; $(\alpha, \beta, \gamma)$ constants which determine the nature and magnitude of dielectric constant. If temperature hysteresis increases under illumination of light, it significantly reduced the effective photo-dielectric constant near ferroelectric phase transition and hence reduces the dielectric constant $\varepsilon_{\mathrm{N}}(T)<\varepsilon(T)$; however, the effects may be opposite if the recharging of deep centers in these ferroelectrics behave in the opposite way. ${ }^{\mathbf{1}}$ In the present investigation with low $\%$ substitution of $\mathrm{Bi}^{3+} / \mathrm{Li}^{1+}$ cations, the dielectric constant increases near the phase transition temperature (Fig. 5); however, a similar situation occurs yet systems show significant decreases in dielectric constant near the ferroelectric phase transition temperatures for higher\% optically active charge centers.

A comparative study of temperature dependent dielectric constant and loss tangent has been carried out on these compositions under dark and illumination of light conditions at $5 \mathrm{kHz}$ probe frequency over a wide range of temperatures (from $25-450{ }^{\circ} \mathrm{C}$ ) as shown in Fig. 5(a)-(f). The enhancement in dielectric constant was observed for composition $x=0.3$, but the magnitude of dielectric constant suppressed for $x=0.4$ and 0.5 under illumination conditions near the phase transition temperature. The change in the magnitude of dielectric constant and Curie points can be understood in context of recharging of photo-active trap centers of charge carriers. The equation determines the change in Curie points under 

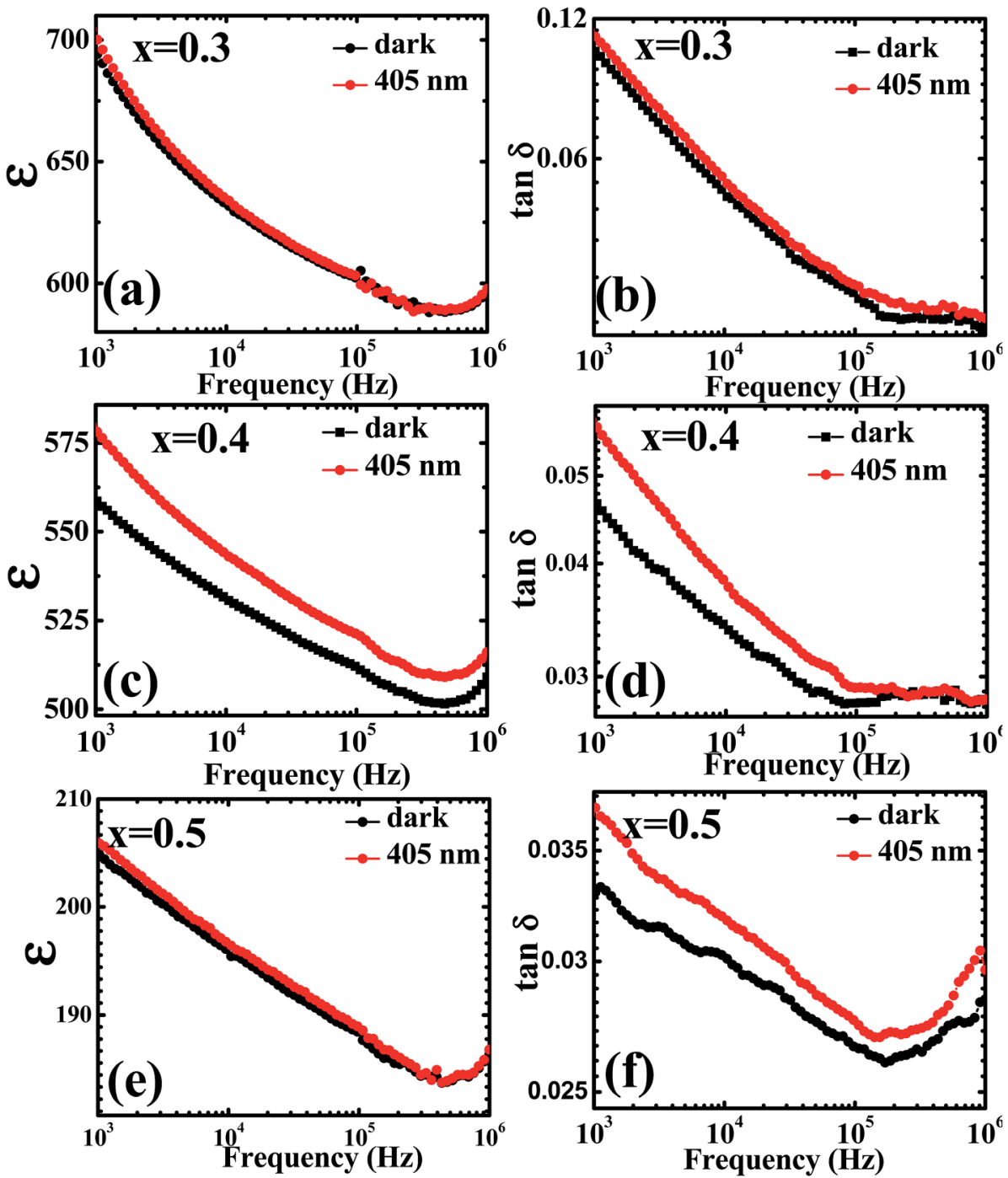

Fig. 4 Room temperature dielectric constant and loss tangent measured for PLBZT in the frequency range of $1 \mathrm{kHz}$ to $1 \mathrm{MHz}$ under dark and illuminated condition of LASER for $x=0.3$ ( $a$ and b), 0.4 ( $c$ and d), 0.5 (e and f).

illumination is as follows: $T_{\mathrm{ON}}-T_{\mathrm{O}}=\Delta T_{\mathrm{N}}=-\frac{C}{2 \pi} a N$ where, $T_{\mathrm{ON}}$ is Curie point in presence of charge carriers $(N)$ under illumination, $T_{\mathrm{O}}$ is Curie point in absence of light assisted charge carriers, $C$ is Curie constant, and $a \cong \frac{\partial^{2} \bar{E}}{\partial P^{2}}$ is a constant obtained by minimizing the free energy. ${ }^{35}$ The enhancement/ decrease in the magnitude of dielectric constant near Curie temperature may be also ascribed to other causes, namely, an intrinsically high polarizability near Curie points due to their ionic displacement or alternatively, morphology features at the interface of the grains/grain boundaries, and with the interface of transparent electrode/electrolytes that modified the polarizability and hence total dielectric properties. The thermodynamics of ferroelectric ceramics indicates that an optodielectric phenomenon is not fully understood based on their microscopic and macroscopic phase transition mechanisms. It is also possible that during ferroelectric phase transition under illumination, large number of photo-generated charge carriers significantly affect the unit cell volume. ${ }^{53}$ The effective dielectric constant has dependence on the presence and concentration of photo generated charge carriers. Note that one can include the extrinsic effects such as Maxwell-Wagner space charge effects, but tangent loss also follows the same behavior, i.e. it significantly decreases near the phase transition temperature under illumination.

(e) Room temperature photo-ferroelectricity, displacement current and PUND analysis

Polarization was measured for PLBZT for $x=0.3,0.4$ and 0.5 on poled electro-ceramics. Fig. 6(a)-(c) show ferroelectric loops measured for $2 \mathrm{~Hz}$ under dark, illumination of a white light source, and monochromatic laser light, since bulk electroceramics do not respond to higher probe frequency. ${ }^{54}$ Correspondingly derived displacement currents are shown in Fig. 6(d)-(f) for the same conditions. A well defined peak in displacement current can be seen for $x=0.3 \& 0.4$ which 

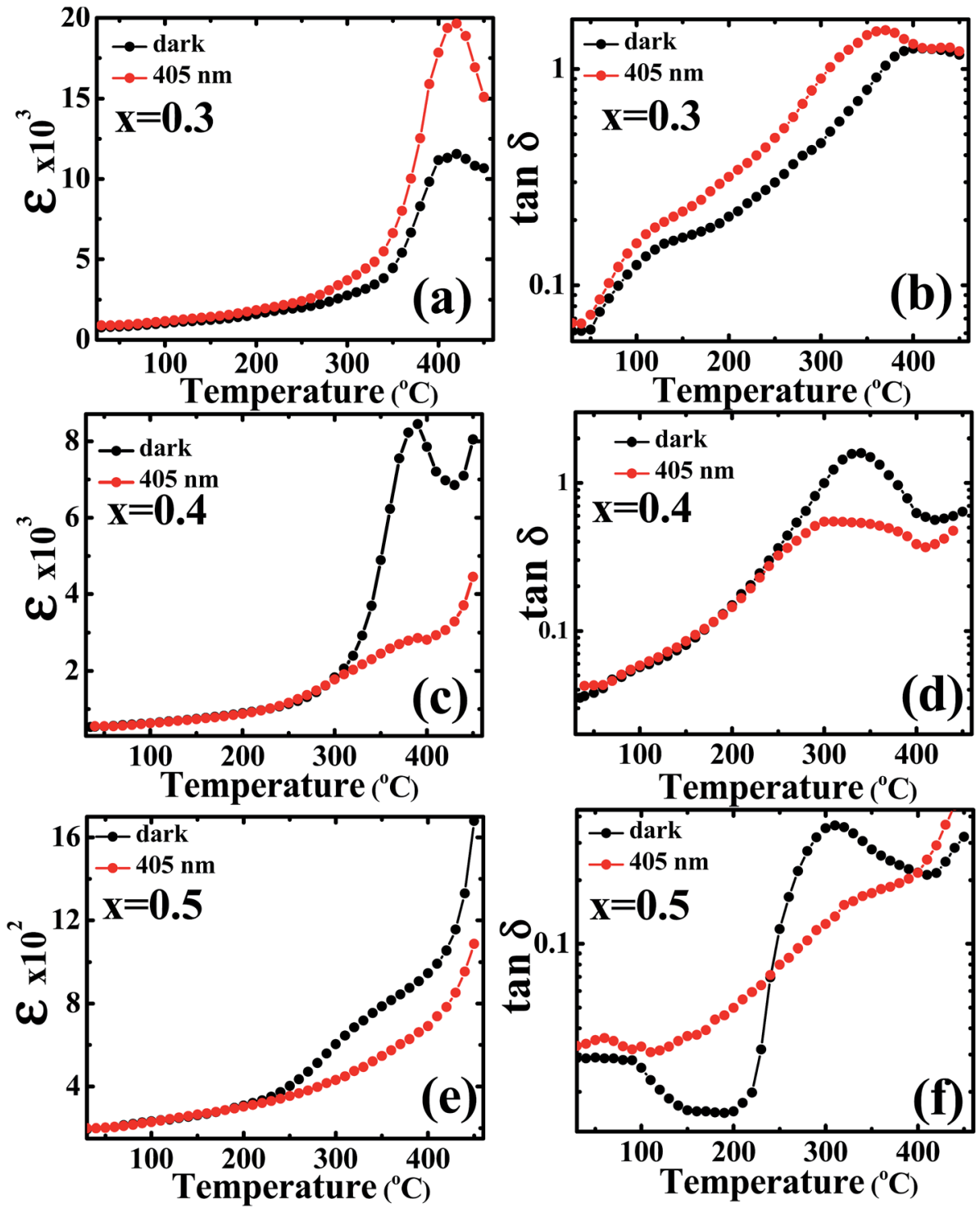

Fig. 5 Dependence on temperature of dielectric constant and loss of PLBZT under dark and illumination of coherent source of LASER $405 \mathrm{~nm} x$ $=0.3(\mathrm{a}$ and $\mathrm{b}), 0.4$ (c and d), 0.5 (e and f) at frequency of $5 \mathrm{kHz}$ in the temperature range of $25-450{ }^{\circ} \mathrm{C}$.

increases under illumination of light; however, for higher compositions it shows only a sharp edge of resistive switching. Under illumination conditions non-equilibrium charge carriers appear which change the electron density and favor the overall enhancement in polarization. The mechanism is associated with the screening of ferroelectric polarization by the charge localized at the levels in the volume and on the surface. This mechanism causes bending of the bands and results in an internal field distribution.

On switching of E-field (electric field) in the light, when the external field reverses its direction, the field strength in the illuminated sections increases, especially near the surface where the screening charge is localized. Non-equilibrium carriers, which redistribute under E-fields applied to the ceramics, influence the overall polarization (electronic: due to change in spectral weight; and ionic: ionic displacement of cations at $\mathrm{A}^{-}$and $\mathrm{B}$-site). We understand that under the influence of white or of monochromatic light the optically active materials absorb photon energy to fill the traps, shift the spectral weight of electronic orbitals, and hence influence the polarization. ${ }^{55-57}$ The enhancement in polarization by the photo-induced charge carriers can be attributed towards the compensation of depolarization field.

Under exposure of white light source the observed polarization $\left(P_{\mathrm{r}}\right)$ increased $50-150 \%$, which may be due to the compensation of the internal depolarization field by photoinduced charge carriers, electronic polarization and ionic shift in various A- and B-site cations. ${ }^{8}$ The polarization also increased by $100-250 \%$ under $405 \mathrm{~nm}$ monochromatic laser light compared to dark polarization, it may be due to energy matching of laser light to that of bandgap of materials. These results suggest anomalous enhancement in spontaneous and remnant polarizations under illumination and may be 

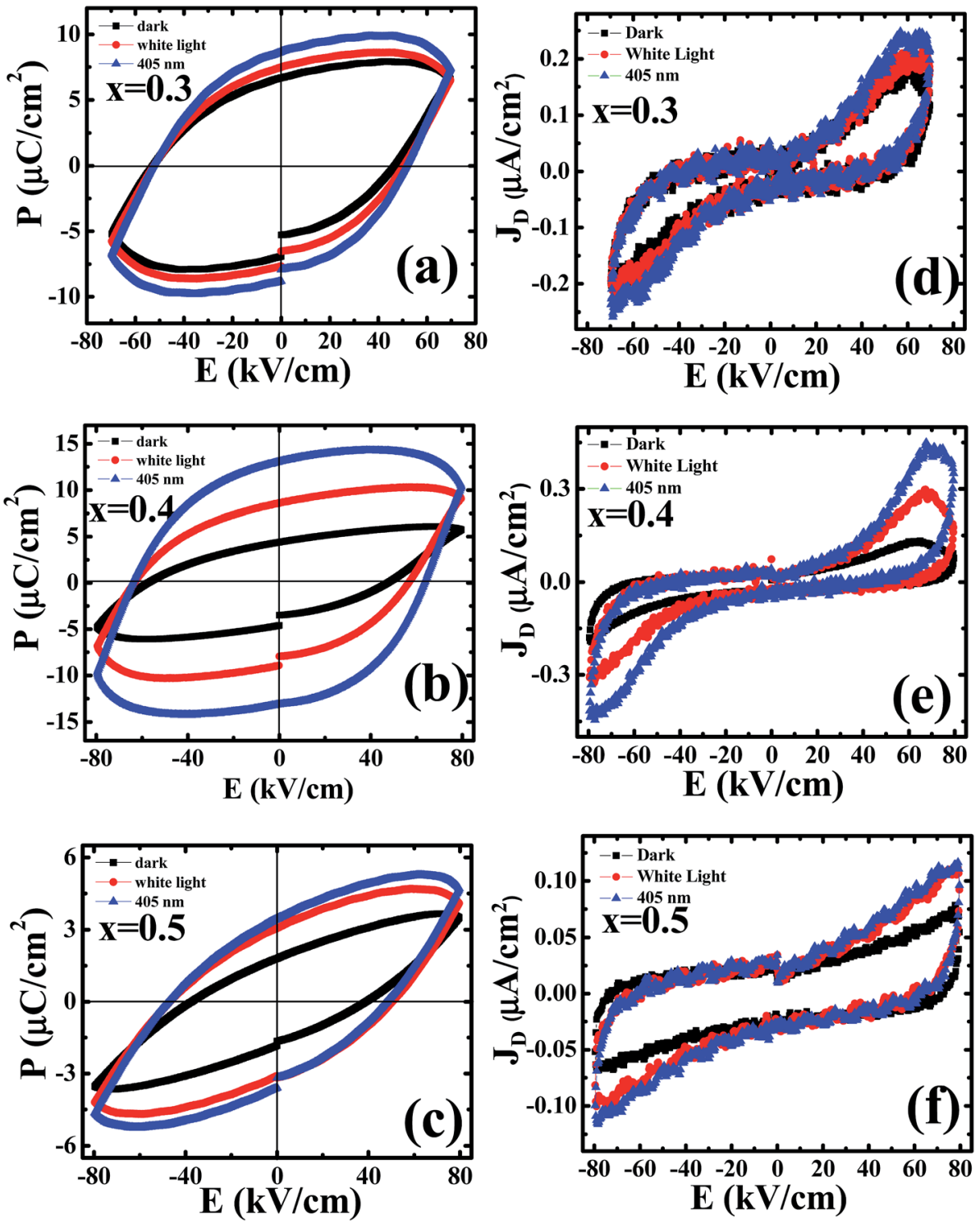

Fig. 6 Ferroelectric polarization measured under three different condition i.e. dark, white light source and monochromatic laser having $405 \mathrm{~nm}$ wavelength for $x=0.3$ (a), 0.4 (b), 0.5 (c). Switching of displacement current derived from ferroelectric polarization loop for $x=0.3$ (d), 0.4 (e), 0.5 (f).

a suitable candidate for devices working on the principle of the switching of displacement currents.

Here we also determine logic characteristics of the PLBZT ceramics using PUND (Positive Up and Negative Down) measurement for ferroelectric memory elements based on a series of five pulses. Fig. 7 shows that PUND measurements were performed at $80 \mathrm{kV} \mathrm{cm}{ }^{-1} \mathrm{E}$-field stress and pulse width at $100 \mathrm{~ms}$ time. The calculation of the switchable polarization as follows: $\mathrm{d} P=P^{*}-P^{\wedge}=$ where $P^{*}=$ (switchable polarization + non-switchable polarization) and $P^{\wedge}=$ (non-switchable polarization); $\mathrm{d} P_{r}=P_{\mathrm{r}}^{*}-P_{\mathrm{r}}^{\wedge}$ where $P_{\mathrm{r}}^{*}=$ (switchable remanent polarization + non-switchable remanent polarization) and $P_{\mathrm{r}}^{\wedge}=$ (nonswitchable remanent polarization); theoretically the values of $\mathrm{d} P$ and $\mathrm{d} P_{\mathrm{r}}$ (experimental values in Table 3 ) in the same range. PUND analysis indicates almost $50-100 \%$ switchable polarization under illuminated conditions for $x=0.3,0.4,0.5$, however among these three systems, $x=0.4$ shows the highest switching under illumination. These results suggest that light illumination not only switches the polarization but also dictates the charge carriers.

\section{(f) Current conduction mechanisms}

Current density $(J)$ versus electric field $(E)$ measurement data in dark and under white light source is shown in Fig. 8(a)-(c). Symmetrical leakage current behaviors were observed for series of compositions $x=0.3$ to 0.5 up to $\pm 60 \mathrm{kV} \mathrm{cm}^{-1}$ E-field stress. A kink in the conduction data near $45 \mathrm{kV} \mathrm{cm}^{-1}$ to $55 \mathrm{kV} \mathrm{cm}^{-1}$ was seen that represents the displacement current switching near the coercive fields. Inset data show leakage current behavior of unpoled electro-ceramics for their respective compositions. After E-poling, nearly saturated and trap-free charge carriers were present in the matrix that also 

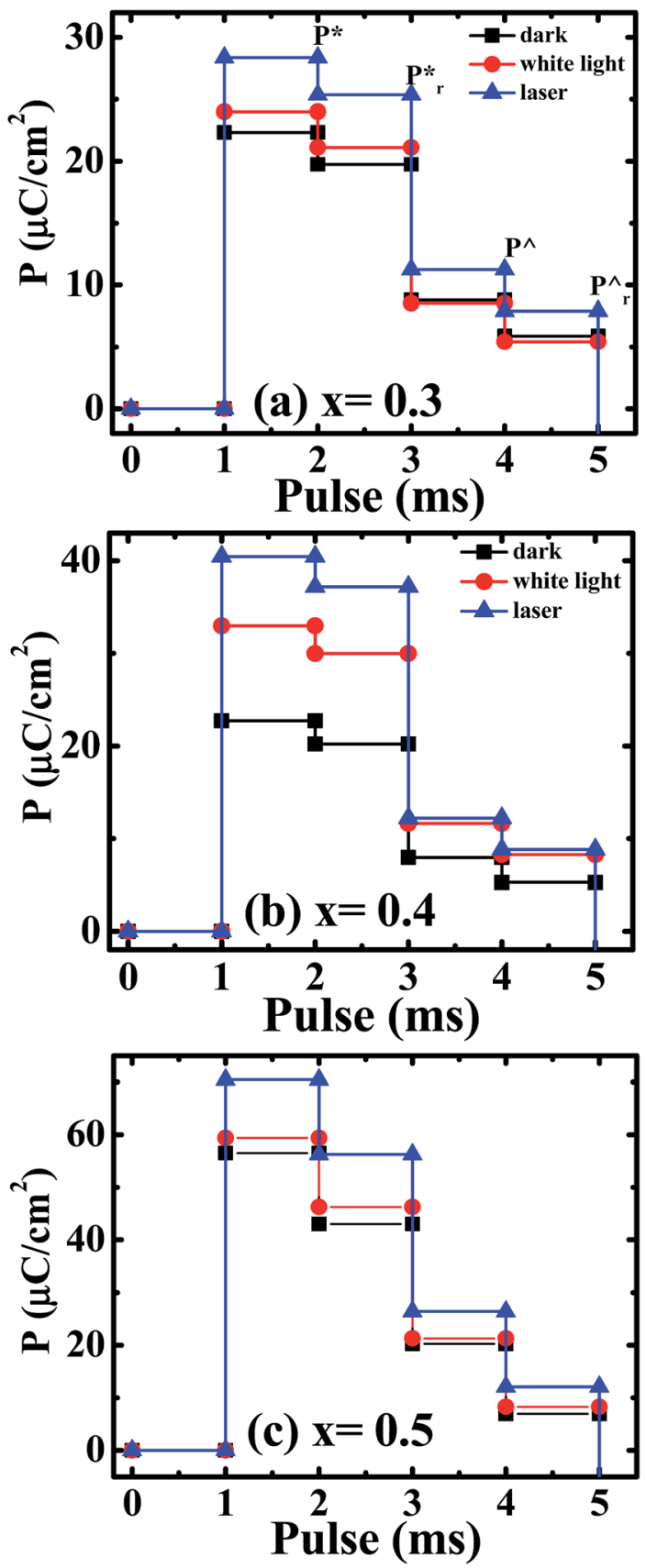

Fig. 7 PUND characteristics for poled ceramics at applied E-field 80 $\mathrm{kV} \mathrm{cm}{ }^{-1}$ and pulse width 100 ms under dark and illuminated conditions for (a) $x=0.3$, (b) $x=0.4$, (c) $x=0.5$.

significantly (at least an order) increased under illumination. These results suggest the development of surface charge carriers near electrode-electrolyte interface and hopping of trapped charges across grain boundaries to the nearest neighbor positions under illumination of light.

In the hope of understanding the mechanism responsible for conduction for PLBZT ceramics, we have considered four main mechanisms, space charge limited conduction (SCLC) mechanisms ${ }^{58}$ Schottky emission (SE) ${ }^{59}$ modified SchottkySimmons model, ${ }^{60}$ and bulk-limited Poole-Frenkel (PF) ${ }^{61}$ These models are commonly used to describe carrier transport in ceramics/ferroelectrics. We fitted $J-E$ data for quantitative analysis of charge transport in matrix and across interface. Depending on the type of charge transport, experimental data should satisfy straight line fits, and the optical dielectric constant of materials can be extracted theoretically from the magnitude of slopes. Therefore we present some set of results analyzed in terms of the different models.

First we have fitted our data for SCLC conduction mechanism where charge carriers are dominated by space charge (either from free or trapped carriers). The current density $(J)$ is expected to have a power-law dependence on applied E-field $(J \propto$ $\left.E^{n}\right) .{ }^{62}$

$$
J_{\mathrm{SCLC}}=\frac{9 \mu_{\mathrm{p}} \varepsilon_{\mathrm{r}} \varepsilon_{0} \theta E^{2}}{8 d}
$$

where, $E$ is bias electric-field, $\varepsilon_{\mathrm{r}}$ is the dielectric constant of ceramics, $\varepsilon_{0}$ is the dielectric constant of free space, $\mu_{\mathrm{p}}$ is carrier mobility, $\theta$ is fraction of injected charge carriers that is free (i.e. not trapped); in trap free limit $\theta=1$.

Fig. 8(d)-(f) show the linear fitting of $\log J$ vs. $\log E$, it provides slopes for $x=0.3,0.4,0.5$ under dark and illuminated conditions for poled ceramics. It can be easily identified two distinct regions, one below coercive fields and above coercive fields. The linear dependence of $\log J-\log E$ plot in both regions satisfy the power-law dependence. Below coercive fields, the values of exponents $(n)$ were found to be in the range of 1.29 to 2, which is compatible with the discrete trap assisted conduction mechanisms. Therefore we believe that trap assisted discrete conduction mechanisms may be one of the dominant factors to control the conduction process in PLBZT ceramics in both conditions. These findings suggest that optically active $\mathrm{Li} / \mathrm{Bi}$ cations at A-site can redistribute the anion (oxygen) vacancies and defects trapped across grains and grain boundaries.

The optical dielectric constants obtained from the slopes of graphs $\ln J$ vs. $E^{1 / 2}$ (pure Schottky model, Fig. 9(a)-(c)) are in the range of 0.3 to 1.9 for $x=0.3$ and 0.4 which provide

Table 3 Switchable and non-switchable polarization form PUND measurement for PLBZT ceramics

\begin{tabular}{|c|c|c|c|c|c|c|c|c|c|}
\hline \multirow{2}{*}{ Conditions } & \multicolumn{9}{|c|}{ Compositions } \\
\hline & \multicolumn{3}{|c|}{$x=0.3$} & \multicolumn{3}{|c|}{$x=0.4$} & \multicolumn{3}{|c|}{$x=0.5$} \\
\hline $\mathrm{d} P\left(\mu \mathrm{C} \mathrm{cm}{ }^{-2}\right)$ & 13.55 & 15.47 & 17.12 & 14.79 & 21.35 & 28.27 & 36.26 & 38.13 & 44.05 \\
\hline $\mathrm{d} P_{\mathrm{r}}\left(\mu \mathrm{C} \mathrm{cm}{ }^{-2}\right)$ & 13.87 & 15.48 & 17.47 & 14.97 & 21.71 & 28.37 & 36.11 & 37.96 & 44.15 \\
\hline
\end{tabular}



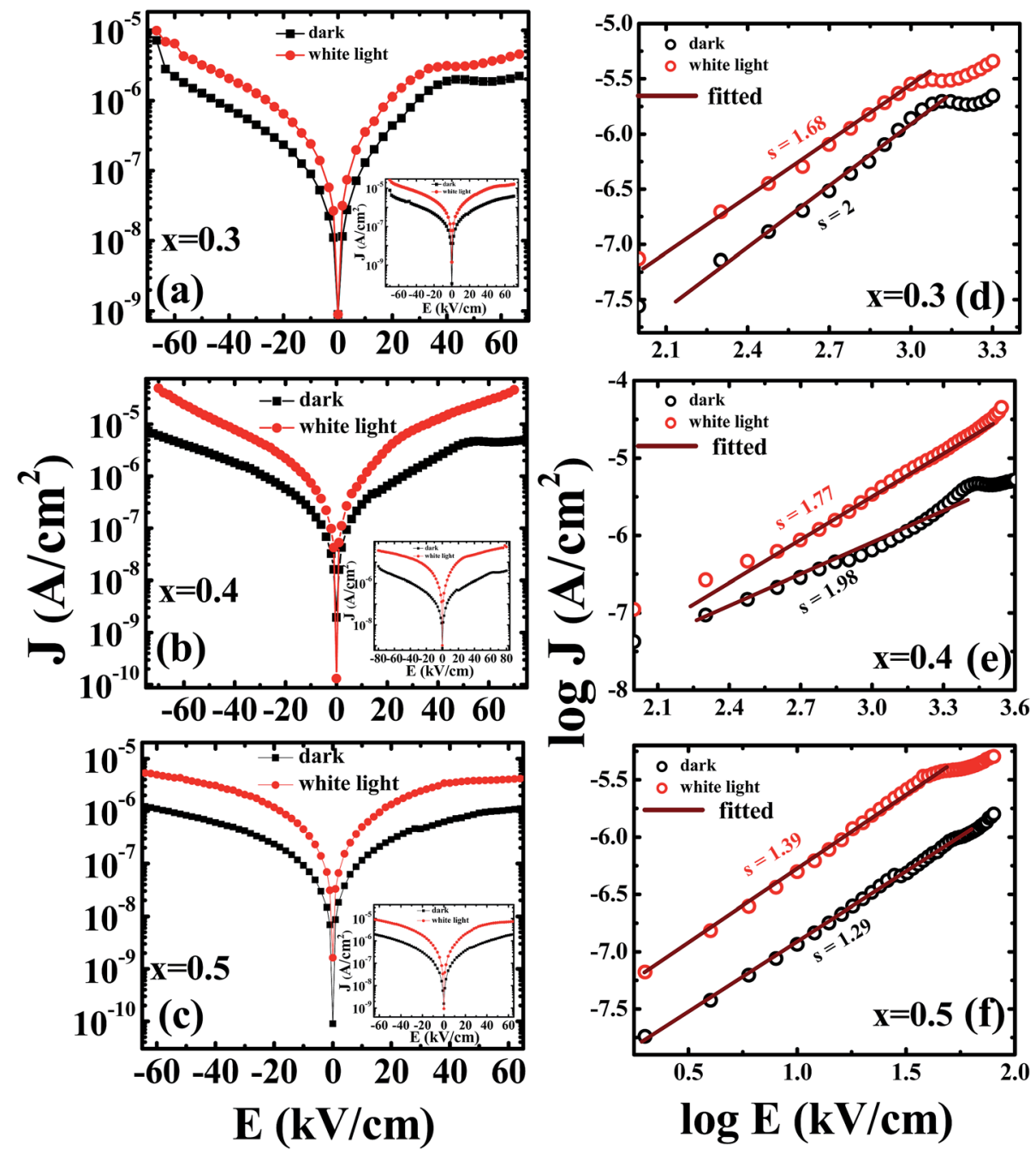

Fig. 8 Leakage current density $(J)$ as function of electric field (E) for PLBZT systems for poled electroceramics (inset unpoled) under dark and illumination of white light source: (a) $x=0.3$, (b) $x=0.4$, (c) $x=0.5$. Conduction mechanisms for PLBZT ceramics studies using $J-E$ characteristics SCLC process for electrically poled electroceramics and fitted with SCLC (log(J) vs. $\log (E))$ plots for (d) $x=0.3,(e) x=0.4,(\mathrm{f}) x=0.5$.

unrealistic values of optical dielectric constant hence ruled out its importance. Later we have fitted our data for SchottkySimmons model and Poole-Frenkel $\left(\beta_{\mathrm{PF}}=2 \beta\right)$ for the determination of optical dielectric constant as shown in Fig. 9(d)-(f) for Schottky-Simmons/Poole-Frenkel model. The observed data linearly fit the Schottky-Simmons/Poole-Frenkel models (most suitable for oxides where charge carriers possess short mean-free paths and bulk process), however, the experimental data for optical dielectric constant of Ti rich PZT only matched with the Poole-Frenkel model with $\left(\xi=1\right.$ and $\left.\beta_{\mathrm{PF}}=2 \beta\right)$, similar results were obtained for thick PZT thin films by Zubko et al. The detail values of optical dielectric constants obtained for Poole-Frenkel model/Schottky-Simmons model are given in the Table 4. One can correctly distinguish between the Poole-Frenkel (PF) ( $\xi$ is nearly 1) model and Schottky-Simmons model ( $\xi$ is nearly 2 ) based on the thickness dependent $I-V$ data and variation of constant $\xi$ from 1 to 2 . It can be easily seen from the Table 4 that with increase in $\xi$ values, the optical dielectric constant values obtained from the slopes of various experimental data deviate from the optical dielectric constant $(\sim 6-8)$ of Ti rich PZT reported in literature ${ }^{63}$ hence ruled out the Schottky-Simmons model (interface limited) and support PF mechanisms (bulk conduction). However, the optical dielectric constant obtained from the slopes for $x=0.5$ compositions favor either Schottky-Simmons model or discrete trap assisted SCLC mechanism. At least for $x=0.5$ samples require further thickness dependent study to reach any further conclusion which is beyond the scope of present investigation.

Schottky emission model:

$$
J(E)=A^{*} T^{2} \exp \left[-e \phi+\frac{e^{\frac{3}{2}} V^{\frac{1}{2}}}{4 \pi d \varepsilon_{0} K}\right] / k T
$$

where $J$ is current density, $V$ is applied voltage, $A^{*}$ is Richardson constant $=4 \pi e m^{*} k_{\mathrm{B}}{ }^{2} / h^{3}$, $e$ is electronic charge, $\varepsilon_{0}$ is permittivity in free space, $K$ is optical dielectric constant, $k_{\mathrm{B}}$ is Boltzmann constant, $T$ is absolute temperature, $\phi$ is barrier height. 

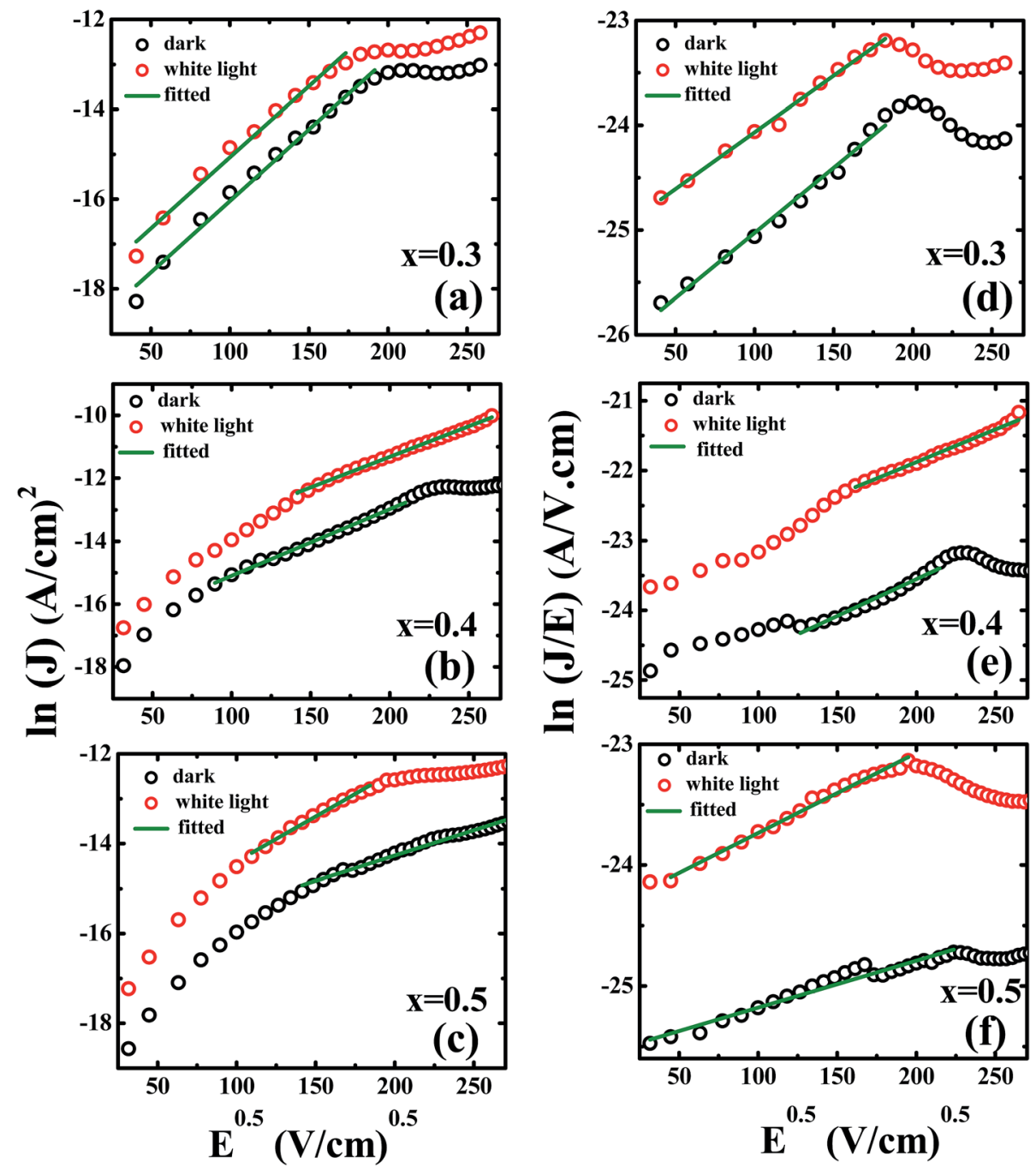

Fig. 9 Leakage current density (J) as function of electric field (E) fitted with Schottky emission (a) $x=0.3$, (b) $x=0.4$, (c) $x=0.5$ and fitted with modified Schottky-Simmons equation/Poole-Frenkel model (d) $x=0.3$, (e) $x=0.4$, (f) $x=0.5$ under dark and illumination of white light.

Table 4 Calculated optical dielectric constant from modified Schottky-Simmons equations

\begin{tabular}{|c|c|c|c|c|c|c|}
\hline \multirow[b]{3}{*}{ Conditions } & \multicolumn{6}{|c|}{ ( $\xi, K, \&$ slopes) } \\
\hline & \multicolumn{2}{|c|}{$\begin{array}{l}\text { Poole-Frenkel model valid for } \xi \\
=1(x=0.3)\end{array}$} & \multicolumn{2}{|c|}{$\begin{array}{l}\text { Poole-Frenkel model valid for } \\
\xi=1(x=0.4)\end{array}$} & \multicolumn{2}{|c|}{$\begin{array}{l}\text { Poole-Frenkel model valid for } \\
\xi=1(x=0.5)\end{array}$} \\
\hline & Dark & White light & Dark & White light & Dark & White light \\
\hline Values of slopes & 0.01245 & 0.0108 & 0.0105 & 0.0093 & 0.0039 & 0.0066 \\
\hline$K(\xi=1)$ & 5.6 & 7.4 & 7.8 & 9.9 & 52 & 19.7 \\
\hline$K(\xi=1.25)$ & 3.5 & 4.7 & 5.0 & 6.3 & 36 & 12 \\
\hline$K(\xi=1.5)$ & 2.4 & 3.2 & 3.4 & 4.4 & 25 & 8.7 \\
\hline$K(\xi=1.75)$ & 1.8 & 2.4 & 2.5 & 3.2 & 18 & 6.5 \\
\hline$K(\xi=2)$ & 1.3 & 1.8 & 1.9 & 2.4 & 14 & 4.5 \\
\hline
\end{tabular}

Schottky-Simmons model:

$J(E)=a E \mu T^{\frac{3}{2}}\left(m^{*} / m\right)^{3 / 2} \exp \left\{-\left(\frac{\phi}{k_{\mathrm{B}} T}\right)+\frac{1}{k_{B} T}\left(\frac{e^{3} V}{4 \pi \varepsilon_{0} K d}\right)^{1 / 2}\right\}$ or

Poole-Frenkel model/Schottky-Simmons model:

$$
\ln (J / E)=\frac{1}{\xi k_{\mathrm{B}} T} \beta \sqrt{E}+\left[\ln C-\frac{q \phi_{\mathrm{s}}}{\xi k_{\mathrm{B}} T}\right]
$$


where $\beta_{3}=\left(e^{3} / \pi \varepsilon_{0} K\right)^{1 / 2}, \xi$ is in the range of 1 and 2, $C=a \mu T^{\frac{3}{2}}\left(m^{*} / m\right)^{3 / 2}, \alpha=3 \times 10^{-4} \mathrm{~A} \mathrm{~s} \mathrm{~cm}^{-3} \mathrm{~K}^{-3 / 2}, J$ is current density, $V$ is applied voltage, $\mu$ is carrier mobility, $m^{*}$ and $m$ are the effective and free electron mass, respectively, $\varepsilon_{0}$ is permittivity in free space, $k$ is Boltzmann constant, $T$ is absolute temperature, $K$ is optical dielectric constant, and $\phi$ is barrier height. For PooleFrenkel model: $\beta_{\mathrm{PF}}=2 \beta$ and $\xi$ is nearly 1 to 1.25 and for Schottky-Simmons model $\xi$ is near to 1.75 to 2 , depending on the microstructure and materials internal properties.

The current conduction mechanisms also depend on the work function of electrodes and their conductivity. We have used a transparent electrode ITO (50 ohm) as top electrode to check the leakage current under the illumination of light. The energy band diagram of ITO/PLBZT/Ag structure suggests well defined Schottky barrier height. The work function of ITO is $4.7 \mathrm{eV}$, PLBZT is (electron affinity $(3.5 \mathrm{eV})+$ bandgap (3.2-3.4 $\mathrm{eV})$ ), and bottom electrode $\mathrm{Ag}$ is $4.8 \mathrm{eV}$. Illumination of light modifies the Fermi energy and populates the conduction band hence effectively reduce the Schottky barrier height and hence effectively increase the charge injection across electrodedielectric interface. The modification of barrier height and development of space-charge in matrix together influence the leakage current under dark and illumination conditions. ${ }^{21}$

The optically active charge carriers remarkably changed the spectral weight of electrons, improve their hybridization, and allow scattering of activated charge carriers at Li/Bi cations. The photo-generated charge carriers are rather uniformly distributed in the samples; even in the case of reversed polarity, illumination enhanced conductivity at least an order of magnitude. This study also highlights the evidence for long lasting generation of photo charge carries and their strong photon-phononelectron coupling. It is evident that considerable amount of photo-carriers generate under illumination of white light near to the top electrode of a materials which drift towards other electrode via grains and grain boundaries (bulk limited) under external biased E-field, during the course of transportation, these carriers fill the traps, compensate the charge carriers across the grains and grain boundaries interface.

\section{Conclusions}

An optically active family of opto-ferroelectrics (PLBZT for $x=$ $0.3,0.4,0.5$ ) has been successfully discovered which shows giant polarization switching under illumination of light. These systems possess tetragonal crystal structure with $P 4 m m$ space group symmetry with increase in in-plane lattice constant (a) and decrease in out of plane lattice constant $(c)$ with increase in $\mathrm{Li} / \mathrm{Bi}$ substitution. The systems with $x=0.4 \& 0.5$ show almost 10-15 ${ }^{\circ} \mathrm{C}$ shift in Curie points towards lower temperature and a significant reduction in the magnitude of dielectric constant under illumination near the ferroelectric phase transition temperature. Interestingly illumination of light also reduced the tangent lost at room temperature and near Curie points, these observations ruled out the possible role of MaxwellWagner effects on dielectric and polarization properties. The polarization and related displacement currents significantly switched under illumination of light depending on the probed frequencies and applied E-fields. The current conduction mainly obeys bulk limited Poole-Frenkel mechanism which provides the optical dielectric constant similar to Ti-rich PZT. The illumination of light changes the spectral weight of electronic orbitals, reorient the polarization, and strengthens the ionic polarization vector in direction applied E-fields resulting a giant switching of polarization under illumination. Hopefully these results guide the ferroelectric family to develop new optically active ferroelectrics for possible uses as electrically WRITE and optically READ non-volatile memory elements, ferro-photovoltaic materials and holographic imaging.

\section{Acknowledgements}

Hitesh Borkar would like to acknowledge the UGC (SRF, Grant No. F.2-61/98 (SA-I)) to provide fellowship to carry out Ph.D. program. Authors would like to thank Prof. R. S. Katiyar (University of Puerto Rico, USA) for XPS measurements and Dr B. P. Singh for Raman experiment. Authors sincerely thank the Director, NPL New Delhi, Dr Ranjana Mehrotra and Dr Sanjay Yadav for their constant encouragement.

\section{References}

1 V. M. Fridkin, Photoferroelectrics, Springer-Verlag, Berlin, 1979.

2 V. K. Wadhawan, Introduction to Ferroic Materials, Gordon and Breach Science Publishers, Amsterdam, 2000.

3 S. Y. Yang, J. Seidel, S. J. Byrnes, P. Shafer, C. H. Yang, M. D. Rossell, P. Yu, Y. H. Chu, J. F. Scott, J. W. Ager, L. W. Martin and R. Ramesh, Nat. Nanotechnol., 2010, 5, 143-147.

4 R. Blinc, G. Tavčar, B. Žemva, D. Hanžel, P. Cevc, C. Filipič, A. Levstik, Z. Jagličić, Z. Trontelj, N. Dalal, V. Ramachandran, S. Nellutla and J. F. Scott, J. Appl. Phys., 2008, 103, 074114.

5 R. Blinc, B. Zalar, P. Cevc, A. Gregorovič, B. Žemva, G. Tavčar, V. Laguta and J. F. Scott, J. Phys.: Condens. Matter, 2009, 21, 045902-045904.

6 R. Blinc, G. Tavčar, B. Žemva, E. Goreshnik, D. Hanžel, P. Cevc, A. Potočnik, V. Laguta, Z. Trontelj, Z. Jagličič and J. F. Scott, J. Appl. Phys., 2009, 106, 023924.

7 K. Yamauchi and S. Picozzi, Phys. Rev. Lett., 2010, 105, 107202-107204.

8 A. L. Kholkin, S. O. Iakovlev and J. L. Baptista, Appl. Phys. Lett., 2001, 79, 2055-2057.

9 A. Bokov and Z. G. Ye, J. Mater. Sci., 2006, 41, 31-52.

10 W. Zhong and D. Vanderbilt, Phys. Rev. Lett., 1995, 74, 25872590.

11 L. M. Belyaev, I. I. Groshik, V. V. Lyakhovitskaya, V. N. Nosov and V. M. Fridkin, JETP Lett., 1967, 6, 16-17.

12 B. I. Sturman and V. M. Fridkin, in The Photovoltaic and Photorefractive Effects in Noncentrosymmetric Materials, Ferroelectricity and Related Phenomena, ed. G. W.Taylor, Gordon and Breach, New York, 1992, vol. 8.

13 M. Dawber, Physics, 2012, 5, 63-66.

14 G. Giovannetti and J. V. D. Brink, Phys. Rev. Lett., 2008, 100, 227603-227604. 
15 K. Kobayashi, S. Horiuchi, R. Kumai, F. Kagawa, Y. Murakami and Y. Tokura, Phys. Rev. Lett., 2012, 108, 237601-237605.

16 C. Alemany, L. Pardo, B. Jimenez, F. Carmona, J. Mendiola and A. M. Gonzalez, J. Phys. D: Appl. Phys., 1999, 32(17), L79.

17 M. L. Calzada, R. Jimenez, A. Gonzalez, J. Garcia-Lopez, D. Leinen and E. Rodriguez-Castellon, Chem. Mater., 2005, 17(6), 1441.

18 V. M. Fridkin and R. M. Magomadov, Pis'ma Zh. Eksp. Teor. Fiz., 1979, 30, 723-726.

19 T. Choi, S. Lee, Y. J. Choi, V. Kiryukin and S. W. Cheong, Science, 2009, 324, 63-65.

20 R. Nechache, C. Harnagea, S. Li, L. Cardenas, W. Huang, J. Chakrabartty and F. Rosei, Nat. Photonics, 2015, 9, 61.

21 X. Z. Zhai, H. M. Deng, W. L. Zhou, P. X. Yang, J. H. Chu and Z. Zheng, RSC Adv., 2015, 5, 82351.

22 J. Wang, J. B. Neaton, H. Zheng, V. Nagarajan, S. B. Ogale, B. Liu, D. Viehland, V. Vaithyanathan, D. G. Schlom, U. V. Waghmare, N. A. Spaldin, K. M. Rabe, M. Wuttig and R. Ramesh, Science, 2003, 299, 1719-1722.

23 J. Wang, C. S. Sandu, E. Colla, Y. Wang, W. Ma, R. Gysel, H. J. Trodahl and N. Setter, Appl. Phys. Lett., 2007, 90, 133107-133113.

24 S. R. Basu, L. W. Martin, Y. H. Chu, M. Gajek, R. Ramesh, R. C. Rai, X. Xu and J. L. Musfeldt, Appl. Phys. Lett., 2008, 92, 091905-091913.

25 A. J. Hauser, J. Zhang, L. Mier, R. A. Ricciardo, P. M. Woodward, T. L. Gustafson, L. J. Brillson and F. Y. Yang, Appl. Phys. Lett., 2008, 92, 222901-222903.

26 G. Arlt, H. Dederichs and R. Herbiet, Ferroelectrics, 1987, 74, 37-53.

27 G. Luchaninov, A. V. Shilinkov, L. A. Shavalov and I. J. Shipkova, Ferroelectrics, 1989, 98, 123-126.

28 C. A. P. Araujo, J. D. Cuchiare, L. D. McMillan, M. C. Scott and J. F. Scott, Nature, 1995, 374, 627-629.

29 Z. G. Zhu, B. S. Li, G. R. Li, W. Z. Zhang and Q. R. Yin, Mater. Sci. Eng., B, 2005, 117, 216.

30 P. W. M. Blom, R. M. Wolf, J. F. M. Cillessen and M. Krijn, Phys. Rev. Lett., 1994, 73, 2107-2110.

31 L. Pintilie, I. Boerasu, M. J. M. Gomes, T. Zhao, R. Ramesh and M. Alexe, J. Appl. Phys., 2005, 98, 124104-124109.

32 L. Pintilie and M. Alexe, J. Appl. Phys., 2005, 98, 124103124108.

33 H. Borkar, M. Tomar, V. Gupta, J. F. Scott and A. Kumar, Appl. Phys. Lett., 2015, 107(12), 122904-122905.

34 B. Noheda, J. A. Gonzalo, R. Guo, S. E. Park, L. E. Cross, D. E. Cox and G. Shirane, Phys. Rev. B: Condens. Matter Mater. Phys., 2000, 61, 8687-8695.

35 J. Rouquette, J. Haines, V. Bornand, M. Pintard, P. Papet and J. L. Sauvajol, Phys. Rev. B: Condens. Matter Mater. Phys, 2006, 73, 224118-224125.

36 J. F. Meng, R. S. Katiyar, G. T. Zou and X. N. Wang, Phys. Status Solidi A, 1997, 164, 851-862.

37 R. S. Katiyar, J. F. Ryan and J. F. Scott, Phys. Rev. B: Solid State, 1971, 4, 2635-2638.

38 G. Burns and B. A. Scott, Phys. Rev. B: Condens. Matter Mater. Phys., 1973, 7, 3088.
39 A. Kumar, N. M. Murari and R. S. Katiyar, Appl. Phys. Lett., 2007, 90, 262907.

40 C. M. Foster, Z. Li, M. Grimsditch, S. K. Chan and D. J. Lam, Phys. Rev. B: Condens. Matter Mater. Phys., 1993, 48, 1016010167.

41 T. Ostapchuk, J. Petzelt, V. Zelezny, S. Kamba, V. Bovtun, V. Porokhonskyy, A. Pashkin, P. Kuzel, M. D. Glinchuk, I. P. Bykov, B. Gorshunov and M. Dressel, J. Phys.: Condens. Matter, 2001, 13, 2677-2689.

42 L. E. Mcneil, M. Grimsditch and R. H. French, J. Am. Ceram. Soc., 1993, 76, 1132-1136.

43 A. Kumar, N. M. Murari and R. S. Katiyar, Appl. Phys. Lett., 2008, 92(15), 152907-152913.

44 A. Slodczyk, P. Colomban and M. Pham-Thi, J. Phys. Chem. Solids, 2008, 69, 2503-2513.

45 M. P. Thi, G. March and P. Colomban, J. Eur. Ceram. Soc., 2005, 25, 3335-3346.

46 L. E. Stoflea, N. G. Apostol, L. Trupin and C. M. Teodorescu, J. Mater. Chem. A, 2014, 2, 14386.

47 K. W. Schroder, H. Celio, L. J. Webb and K. J. Stevenson, J. Phys. Chem. C, 2012, 116, 19737-19747.

48 S. U. Awan, S. K. Hasanain, M. F. Bertino and G. H. Jaffari, J. Phys.: Condens. Matter, 2013, 25, 156005.

49 M. K. Kuneva and V. I. Krastev, J. Mater. Sci.: Mater. Electron., 2000, 11, 629.

50 H. Borkar, V. Rao, S. Dutta, A. Barvat, P. Pal, M. Tomar, V. Gupta, J. F. Scott and A. Kumar, J. Phys.: Condens. Matter, 2016, 28, 265901.

51 M. Nagoshi, T. Suzuki, Y. Fukuda, K. Ueki, A. Tokiwa, M. Kiruchi, Y. Syono and M. Tachiki, J. Phys.: Condens. Matter, 1992, 4, 5769-5781.

52 A. Kumar, D. Barrionuevo, N. Ortega, A. K. Shukla, S. Shannigrahi, J. F. Scott and R. S. Katiyar, Appl. Phys. Lett., 2015, 106(13), 132901.

53 K. T. Butler, J. M. Frost and A. Walsh, Energy Environ. Sci., 2015, 8, 838-848.

54 J. F. Scott, J. Phys.: Condens. Matter, 2008, 20, 021001-021002.

55 L. Pintilie, I. Vrejoiu, G. Le Rhun and M. Alexe, J. Appl. Phys., 2007, 101, 064109-064118.

56 J. Robertson, W. L. Warren and B. A. Tuttle, J. Appl. Phys., 1995, 77, 3975-3980.

57 D. Dimos, W. L. Warren, M. B. Sinclair, B. A. Tuttle and R. W. Schwartz, J. Appl. Phys., 1994, 76, 4305-4315.

58 B. Nagaraj, S. Aggarwal, T. K. Song, T. Sawhney and R. Ramesh, Phys. Rev. B: Condens. Matter Mater. Phys., 1999, 59, 16022-16027.

59 S. M. Sze, Physics of Semiconductor Devices, Wiley, New York, 2nd edn, 1981.

60 J. G. Simmons, J. Appl. Phys., 1964, 35, 2472-2481.

61 P. Zubko, D. J. Jung and J. F. Scott, J. Appl. Phys., 2006, 100, 114113-114117.

62 R. H. Tredgold, Space Charge Conduction in Solids, Elsevier Publishing Company, Amsterdam, 1966.

63 (a) Z. Huang, X. Meng, P. Yang, Z. Zhang and J. Chu, Appl. Phys. Lett., 2000, 76, 3980-3982; (b) J. Robertson and C. W. Chen, Appl. Phys. Lett., 1999, 74, 1168-1170. 